This item was submitted to Loughborough's Research Repository by the author.

Items in Figshare are protected by copyright, with all rights reserved, unless otherwise indicated.

\title{
Priorities for researching 'race', ethnicity and racism in sports coaching and recommendations for future practice
}

PLEASE CITE THE PUBLISHED VERSION

http://www.routledge.com/9780367426699

\section{PUBLISHER}

Routledge

VERSION

AM (Accepted Manuscript)

\section{PUBLISHER STATEMENT}

This is an Accepted Manuscript of a book chapter published by Routledge in Race, Ethnicity and Racism in Sports Coaching on 6 Jul 2020, available online: http://www.routledge.com/9780367426699

\section{LICENCE}

CC BY-NC-ND 4.0

\section{REPOSITORY RECORD}

Lusted, Jim, Steven Bradbury, and Jacco Van Sterkenburg. 2020. "Priorities for Researching 'race', Ethnicity and Racism in Sports Coaching and Recommendations for Future Practice". Loughborough University. https://hdl.handle.net/2134/12473477.v1. 
'Race', Ethnicity and Racism in Sports Coaching

Bradbury, S., Lusted, J. and Van Sterkenburg, J.

Chapter 14: Priorities for researching 'race', ethnicity and racism in sports coaching and recommendations for future practice

\section{Jim Lusted, Steven Bradbury and Jacco van Sterkenburg}

This purpose of this chapter is two-fold. Firstly, it begins by outlining potential priorities for future research into the field of 'race', ethnicity and racism in sports coaching. In doing so, it draws on the work of several authors in this collection to call for a multi-level analysis in proposing future research which considers the complex interplay between the macro (societal), meso (organisational) and micro (individual) level of racialised relations in sports coaching. Secondly, its draws together the key recommendations from the chapters in this collection to outline some prospective priorities for addressing racialised inequalities and establishing a more diverse and equitable environment in sports coaching. In doing so, it calls on key stakeholders in sports to develop and implement a multi-level and holistic approach, incorporating; robust policy interventions, education and training programmes, and enhanced opportunities for networking and the development of social capital amongst minoritised sports coaches.

\section{Introduction}

This book is the first comprehensive collection of academic work focusing specifically on the ways in which 'race', ethnicity and racism operate and are experienced in the field of sports coaching. The chapters featured in this collection reflect the research of established and 
emerging scholars drawn from a range of national contexts across three continents. These scholars have utilised quantitative and qualitative methodologies to generate original empirical data and/or have drawn on and applied a range of distinct but overlapping theoretical and conceptual approaches in their analysis. These efforts have helped to fulfil the original intention to bring together a series of insightful scholarly contributions to this relatively marginalised field of study into one volume. This collection is also well-timed. There is increasingly popular interest in the topic and in recent years some high-profile policy developments and targeted positive action interventions designed to diversify the sports coaching workforce in some sports across the globe have emerged. In this respect, it is hoped that this collection will encourage and empower other scholars, practitioners and students to engage in and with further critical research of this kind in order to extend the diversity of empirical focus and the breadth of theoretical understanding of racialised inequalities in sports coaching.

This concluding chapter begins by presenting some of the editors' observations as to the potential priorities for future research in this important field of enquiry. In doing so, it focuses on issues of consideration at the macro (societal), meso (organisational) and micro (individual) level of the analysis of racialised relations in sports coaching. The chapter also brings together and develops further some of the key recommendations offered by contributing authors in each of the chapters in this collection. In doing so, the editors outline some potential prospective priorities for key stakeholders in addressing racialised inequalities and establishing a more diverse and equitable environment in sports coaching.

\section{Priorities for researching 'race', ethnicity, and racism in sports coaching}

Our chapters represent an increasing breadth and complexity of the types of research questions being asked about the influence and impacts of ideas around 'race', ethnicity and 
racism in sports coaching contexts. The collection is themed around three key areas of investigation; (i) representation and racialised barriers in sports coaching, (ii) racialised identities, diversity and intersectionality in sports coaching, (iii) and formalised racial equality interventions in sports coaching. This impressive scope of subjects highlights the growing scholarly attention that the field of 'race', ethnicity and racism in sports coaching is now receiving and the variety of issues being considered and examined. So what might be the priorities for future research of this kind? We take a lead in this respect from Cunningham's call in Chapter 1 for a multi-level approach to the examination of racialised inequalities in sports coaching which incorporates scholarly analysis at the macro (societal), meso (organisational) and micro (individual) level. As Cunningham states, there are important insights to be gained from adopting such a multi-level approach:

Multilevel models make explicit the societal (macro-level), organizational and group influences (meso-level), and individual factors (micro-level) that can shape access to and experiences in sport. When scholars and practitioners fail to consider factors at multiple levels of analysis, they necessarily take for granted elements that could influence the phenomenon of interest.

(Cunningham 2020: add page number)

To elaborate, at the macro-level, we are broadly referring to the structural conditions that underpin social arrangements; the meso-level refers to the organisational and institutionalised manifestations of these structural conditions, whilst at the micro-level the empirical focus is on the individual, or groups of individuals and their own lived experiences and embodiment of such structural and institutionalised arrangements. This multi-layered way of thinking about social analysis was also drawn on by Bradbury in this collection (Chapter 2) in his succinct overview of previous studies on 'race', ethnicity, racism and sports coaching: 
At the macro-level, scholars have alluded to constraints engendered by the broader political and legislative climate and the role of powerful internal and external stakeholders in the coach hiring process. At the meso-level, they have identified dominant monocultural processes and cultures and the existence of conscious and unconscious bias within the senior decision-making tiers of sports organisations. Further, at the micro level, they have drawn attention to the impact of structural and institutional constraints on inhibiting opportunities for minoritised groups to develop the requisite network capital to engender upward social mobility as coaches.

(Bradbury 2020: add page number)

We offer below some possible priorities to guide future research in this field across all three of these proposed levels of analysis.

\section{Macro- (societal) level analysis: the racialised social conditions of sports coaching}

The majority of previous research in this field - including that which is featured in this collection - has acknowledged the influence of the broader social conditions in which sports coaching operates, to help make sense of the past, current and future picture of racial diversity amongst the coaching workforce. For example, in this collection, the work of Bradbury (Chapter 2), Heim, Corthouts and Scheerder (Chapter 3), Van Sterkenburg (Chapter 4) and Kilvington (Chapter 5) has each drawn attention to the deeply racialised socio-historical and contemporary national political contexts in Europe in which the experiences of minoritised ${ }^{1}$ football coaches have been 'played out'. Similarly, drawing on the US context, the contributions of Cunningham (Chapter 1) and Olushola-Ogunrinde and

\footnotetext{
$1 \quad$ As outlined in the Introduction of this collection, the term 'minoritised' is used in this chapter to reflect an understanding of 'minority status' as a socially constructed process (rather than as an entity) which takes place in specific social, economic and political contexts over time and which has resulted in 'minoritised groups' having less power or representation compared to other (White) groups in society.
} 
Carter-Francique (Chapter 9) have alluded to a series of embedded structural, representational and political barriers at the macro (societal) level and their deleterious impacts in constraining the career progression of minoritised coaches across a range of professional and college sports-based settings.

Our authors have also drawn on a range of distinct but overlapping theoretical and conceptual approaches to help articulate the nature and formation of these racialised structural conditions and their significance in shaping organisational arrangements and individual behaviours. In particular, many authors have drawn on the theoretical insights of Critical Race Theory (CRT) to illustrate the exclusionary impact of dominant liberal ideologies of colourblindness, race neutrality and meritocracy - the latter being particularly influential in sporting cultures. Indeed, these authors owe a debt of gratitude to pioneer advocates of CRT and its application to sport - notably through the work of Hylton $(2005,2009,2010)$ - to the extent that CRT has now arguably become the dominant theoretical lens to examine issues of 'race', ethnicity and racism in sport (and sports coaching).

However, at the same time, we also see room to examine more closely the specific nature and influence of the racialised structural conditions proposed by the CRT approach. Amongst others, Cole (2017) and Cabrera (2018) have pointed to some of the limitations of CRT explanations that we might reasonably seek to problematise in future studies in sports coaching and encourage a more eclectic mix of macro-level explanations. The former claims that CRT approaches can underplay the influence of capitalism and social class in shaping the racialised conditions of our times, favouring instead neo-Marxist ideas and concepts (Cole 2017). Cabrera on the other hand argues that the broad CRT framework contains some theoretical 'holes' that require a more explicit racial theory to be articulated, making the case for a critical theory of racism centred on hegemonic whiteness (Cabrera 2018). 
A more dynamic approach to macro-level analysis might also help provide new insights into the relative rigidity or permeability of the societal structures that underpin racism and racialised exclusion in sports coaching. This may also open up the extent to which there exists commonalities and differences across national contexts with broadly comparative or markedly different racial histories. Further, a more detailed focus on this score might also elicit useful scholarly insights as to the extent to which such conditions have been altered and what factors have contributed to such change. We may then be in a stronger position to assess the impact these changes have had in enabling a space for progressive movements and interventions such as the adoption of the 'Rooney Rule' to emerge. Looking forward, what might these structural conditions need to consist of and how can they reasonably be modified at the macro societal level to open up a space within which to better facilitate more inclusive coaching environments and a more equitable coaching workforce? Similarly, to what extent are these structural conditions changeable by local interventions within sport? As we will discuss later, one of the key recommendations from our chapters is the need for future interventions to address these structural arrangements. The challenge for future research is to further our understanding of how such conditions emerge and are sustained so we can propose suitable ways of re-shaping them.

Whilst there is a need to further our understanding of the specific conditions that shape our sports, we must also remind ourselves that we cannot separate the sporting experience from its wider societal context. Indeed, there is much more we can do to make analytical connections between the structures of sport and wider macro social, political, economic and cultural processes. In current times we are witnessing extremely rapid changes that are significantly challenging our previous assumptions about the world. Fukuyama's claim of the 'end of history' (2006) - an enduring victory for liberal democracy - seems significantly wide of the mark following recent global political events. The US election victory of Donald 
Trump in 2016, the referendum vote in the UK to leave the European Union in the same year, and electoral gains for right-wing political groups across many parts of the world such as Hungary, the Netherlands, Brazil and India have changed the geo-political landscape considerably in recent years. For a longer period of time, the hegemonic control of neoliberal free-market capitalist ideology across large parts of the world has also significantly shaped the structural conditions within which sports operate. The term 'racial neoliberalism' has been used by a range of authors (for example Goldberg 2009, Kapoor 2013, Burdsey 2014) to explore how this broader neoliberal project is also one of 'hidden' racialisation; one that claims on the one hand that ideas of 'race' are meaningless, whilst on the other hand relying upon more subtle, nuanced forms of racialised exclusion to maintain White privilege. The emerging technological revolution and the rise of the information age (Castells 1997) and increasing dominance of social media are shifting these conditions further. It is clearly evident that these macro-level changes have influenced sport more broadly; the \#blacklivesmatter movement in the US, overtly supported by now-ostracised Black NFL quarterback Colin Kaepernick (Boykoff and Carrington 2019), the rising racist abuse on social media of Black professional footballers in England (Farrington et al. 2015), and the increasing confidence of the far-right (re)emerging among sport fan groups across countries including Russia (Glathe 2016) and Italy (Testa and Armstrong 2010). These incidents remind us of the influence that macro socio-political changes can have on the nature of racism and racialised exclusion in sport, and will continue to have in debates around the racial diversity of sports coaches in years to come. 


\section{Meso- (organisational) level analysis: the institutionalised racialisation of sports coaching}

All of the chapters featured in this collection focus their attention in one form or another at the meso organisational level of sports coaching. For example, the contributions of Cunningham (Chapter 1), Bradbury (Chapter 2), Van Sterkenburg (Chapter 4), Kilvington (Chapter 5) and Olushola-Ogunrinde and Carter-Francique (Chapter 9) all allude to the tendencies of sports organisations across a range of national contexts to operate a series of racially closed networks-based approaches to coach recruitment. These organisational processes are argued to constitute a form of institutionalised access discrimination which has restricted opportunities for minoritised coaches and contributed to sustaining patterns of homologous reproduction in the sports coaching workforce. Further, the contributions of Duru (Chapter 11), Cowell (Chapter 12) and Conricode and Bradbury (Chapter 13) have focused directly on specific institutions such as National Governing Bodies of sports in their discussions, and have sought to outline and evaluate the legislative development, operational effectiveness and measurable impacts of policy interventions designed to address racialised inequalities in sports coaching.

Whilst the work in the aforementioned chapters has helped us to assess the ways in which ideas around 'race' and processes of racialisation are institutionalised within sports organisations, particularly in relation to the recruitment and development of coaches, there is much more to be explored here. A deeper critical examination of organisational responses to the lack of diversity in sports coaching is needed to help further our understanding of how such responses have come about, the nature of such responses and the likely impact that they may (or may not) have. What are the key drivers in the formation of these policies and interventions aiming to diversify the coaching workforce? How exactly have they been developed? To what extent do they reflect the wider 'liberal' discourse of equal opportunities 
or seek to challenge and disrupt the dominant organisational structures and cultures of whiteness and White privilege that have been discussed by many authors in this collection? By interrogating more fully the motivations and drivers behind such initiatives we can help create a better picture of how they are likely to be received by the wide range of stakeholders involved including minoritised coaches themselves, as well as increasing our understanding of the shape and scope of barriers that might be encountered during their implementation. This critical interrogation of organisational responses to the under-representation and racialised experiences of minoritised coaches can also help to make sense of the evident resistance that exists towards the need for and implementation of equality policies in sport (Spaaij et al. 2018, 2019). Moreover, such policies have been criticised by some scholars as adopting a bureaucratic audit or 'tick box' approach to promoting equality which focuses primarily on the formalisation of statements and providing paper evidence of compliance over meaningful action and change (Dwight and Biscomb 2018, Lusted 2014, Shaw 2007, Spracklen et al. 2006). We should therefore caution our acceptance of the often celebratory rhetoric of these types of policy initiatives, along with what can be quite ambitious claims made about the likely impact they may have. This call for a more critically cautious approach is borne out by the now relatively mature policy history of equality initiatives in sport (over 30 years in some parts of the world such as the UK, Australia and Canada) that has, in many respects, failed to significantly alter the diversity of the sports coaching workforce (and leadership positions more broadly) or establish more inclusive organisational structures, processes and cultures (Doherty et al. 2010).

Elsewhere, more attention is beginning to be paid to the influence of these meso-level processes in shaping such policies and also the potential role that organisational cultures can have in perpetuating inequalities, often as a direct result of their 'display' of work around inclusion. This phenomenon has been termed 'non-performing inclusion' by Ahmed (2012), 
whose influential work more broadly on diversity work in organisations has begun to be taken up by other sport scholars (Bury 2015, Hylton 2015, Spaaij et al. 2018, 2019, see also Conricode and Bradbury in Chapter 13 of this collection). In her analysis of equality and diversity activities in Higher Education institutions in the UK and Australia, Ahmed highlights the ways that such activities often lead to very little output in the way of institutional change. Actions such as the writing of race equality plans, policies and charters take up a large part of this 'diversity work' but actually require very little action on the part of organisational actors, hence the concept of 'non-performing'. Similarly, Bury's (2015) review of anti-homophobia campaigns led by The English Football Association points to the use of strongly-worded policies and statements rarely being complemented by actions, particularly those that require internal organisational change.

Relatedly, Ahmed (2007) has also pointed to the adoption of the term 'diversity' as not simply a matter of semantics but representing a more considered shift away from ideas of social equality, equity and justice. The latter, arguably more 'radical', approach had characterised previous policies in the field that were seen to have failed or had minimal impact. The concept 'diversity' represents more of a liberal approach whereby the goal is to acknowledge and celebrate difference - such as racialised or ethnic differences - rather than explicitly scrutinise or challenge existing organisational cultures or seek internal change (Lusted 2017). Diversity is also seen to represent the business case for equality, i.e. that claims are made about an increasingly diverse workforce aligning with wider business goals related to efficiency, competitiveness or corporate social responsibility (Lusted 2013, Noon 2007). This organisational tension between interpretations of social equality, and the language used to represent the preferred approach, needs closer examination within sport settings, and within the interventions formed by sports organisations that claim to tackle racialised inequalities in coaching. Taken together, this body of critical work highlights the 
scholarly and practical value in examining more closely the organisational responses to the under-representation and racialised experiences of minoritised sport coaches. This will allow us to reflect in a more critical way the underlying reasons for their adoption, the ideas behind the approaches they are advocating and enable a better understanding of their impact and the reasons behind their successes - limited or otherwise.

\section{Micro- (individual) level analysis: testimonies from the racialised side-lines}

Many of the chapters in this collection offer vivid accounts of the direct experiences and effects of racism and racialised exclusions and the ways in which ideas of 'race' gain credence in sports coaching settings. For example, the contributions of Bradbury (Chapter 2), Van Sterkenburg (Chapter 4), Kilvington (Chapter 5), McDonald and Spaaij (Chapter 6), Light and Evans (Chapter 7), Rankin-Wright and Hylton (Chapter 8), Olushola-Ogunrinde and Carter-Francique (Chapter 9), Knoppers and De Haan (Chapter 10) and Conricode and Bradbury (Chapter 13) all draw on the first-hand or secondary accounts of minoritised coaches and players as a means of moving beyond majoritarian stories and master narratives (Solórzano and Yosso 2002) to illuminate and render visible the historically subjugated testimonies and experiential narratives of minoritised groups in sports coaching contexts. We should recognise here the generous time and energy given by all of the participants from the studies featured in this collection. This type of evidence remains crucial to examining the lived experiences of processes of racialisation.

In order to interrogate the underlying mechanisms behind the racialised exclusions expressed by participants in this collection, many of the authors have connected the lived experiences of minoritised coaches to the range of concepts, theories and explanations that operate more broadly at the meso and macro level of analysis. Further examination in that direction is necessary to tie everyday practice and experience to wider racialised structures and to better 
understand how wider discourses which circulate in sport and sports coaching contribute to regulating conduct and constructing identities and subjectivities in the field. This is important in helping to develop a more layered understanding of the ways in which ideas around 'race' are played out within the interactional spaces of sports coaching and with regard to the manifestation, negotiation and impacts of racism and racialised micro-aggressions on minoritised coaches in these settings (Gearity and Henderson Metzger 2017). In addition, in unpacking some of the more problematic assumptions embedded in debates around cultural diversity and organisational effectiveness in sports coaching (and in leadership more broadly), which in laudably seeking to promote the value and benefits of multi-culturalism might also draw unconsciously on broader racialised narratives which essentialise the skills and abilities of minoritised coaches and have unintended consequences in sustaining patterns of occupational segregation in the sports coaching workplace. It also means that a dual research focus is important in which analysis at the individual level is combined with a scrutiny of those in positions of power and control who play a central role in creating and/or reproducing a sports coaching culture where - as many chapters in the book have shown - White people are privileged whilst minoritised people generally find themselves in a disadvantaged position. We need to know much more about how such systemic discriminatory practices are internalised, reproduced and protected if we are to come up with ways of dismantling the stranglehold of these racialised power relationships in sports coaching contexts. Further, as has been argued by Rankin-Wright and Hylton (Chapter 8), Olushola-Ogunrinde and Carter-Francique (Chapter 9) and Knoppers and De Haan (Chapter 10) in this collection, much more research is needed (and needs to be supported) which draws on and foregrounds the silenced voices and marginalised experiences of black women sports coaches and which seeks to locate these intersectional oppressions 
within the context of institutionally embedded power relations at the organisational and societal level.

One key concept to better grasp the interplay between the individual micro level and wider structures is whiteness. In particular how the processes and discourses of whiteness strengthen and (re)produce inclusion and exclusion at the individual level along racial and ethnic lines. As some authors have already stated (Cabrera et al. 2017), whiteness is a complex concept; it is context-specific and quite hard to define in precise terms. This difficulty to pinpoint whiteness, and to identify precisely how it operates, may actually be an important reason why it is so effective in continuing to structure a range of societal and sporting contexts (Omi et al. 1994 in Cabrera et al. 2017). The core of our understanding of whiteness in this edited collection (shared explicitly by many authors in this book) is of whiteness as a powerful structural and cultural practice through which hierarchical systems of racial domination have become effortlessly created, sustained and reproduced over time (Delgado and Stefancic 2012, Giroux 1997). Further, whiteness can be understood as a powerful discourse which privileges White people whilst simultaneously marginalising minoritised groups on a structural basis (Hylton, 2009). As has been argued by Bradbury (Chapter 2), Van Sterkenburg (Chapter 4) and McDonald and Spaaij (Chapter 6) in this collection, the discourse of whiteness is evident within the sports coaching context through the promulgation of powerful racialised ideologies and meanings which have enabled the signification of human characteristics in such ways as to problematise the assumed attributes of minoritised coaches (and players). For example, when key decision makers consider members of the White majority ethnic group 'by default' as the 'norm' and as possessing desirable traits for positions of coaching/management (e.g. leadership, perseverance, intellect), whilst associating members of minoritised groups with athletic performance-based 
roles through an emphasis on physicality rather than mentality, and emotionality rather than rationality.

For future critical research and related anti-racist action in the field of sports coaching, there is a real challenge in intervening in this discourse. Whiteness usually remains hidden below the surface as those who use and benefit from it often draw on a rhetoric of colour-blindness that safely accommodates the ideals of equality and diversity. Colour-blindness can be seen as an ideology that frames situations of racism and racial inequality as completely unrelated to 'race', 'providing raceless explanations for all sort of race-related affairs' (Bonilla-Silva 2015, p. 1364). In this respect, colour-blind ideologies fail to recognise racism unless manifest in its most egregious forms and actively seek to naturalise the consequences of racialised inequalities as resulting from individual and cultural deficit rather than as being created and sustained by the practices of dominant individuals and institutions (Bonilla-Silva 2010). In doing so, colour-blind ideologies tend to strip the realities of success or failure from the deeply racialised contexts in which they occur and consequently legitimate opposition to race-conscious equality policies designed to redress historical racialised imbalances at a societal and sporting level. This is reflected in the contributions of Van Sterkenburg (Chapter 4) and Conricode and Bradbury (Chapter 13) in this collection when minoritised football coaches in the Netherlands and senior staff in English football club academies adhered to (neo)liberal notions of meritocracy, race neutrality and colour-blindness in justifying their explicit resistance towards positive action measures such as the 'Rooney Rule' or mandatory code of coach recruitment. Given the interplay between wider exclusionary discourses on the one hand and individual experiences of minoritised coaches on the other, future research in this field might seek to provide more insights into and challenge the self-evident character of whiteness and colour-blindness in the domain of sports coaching. 


\section{Priorities for addressing racialised inequalities in sports coaching}

Having laid out some suggested priorities for future research in this field, this section turns to some of the key recommendations offered by contributing authors in each of the chapters in this collection. In doing so, it is designed to offer a general account of what our authors feel needs to be prioritised in order for future policies and interventions to be effective in addressing racialised inequalities in sports coaching and in establishing a more diverse coaching workforce. The various recommendations have been collated into four key themes: (i) establishing a multi-level and holistic approach to tackling racialised inequalities in sports coaching, (ii) developing and implementing robust policy interventions, (iii) developing and implementing education and training programmes, (iv) and enhancing networks, social capital and opportunities for minoritised coaches.

(i) Establishing a multi-level and holistic approach to tackling racialised inequalities in sports coaching

A number of authors in this collection have alluded to the limitations of previous and current policies and interventions aimed at diversifying the sports coaching workforce. In the main, the critique here has been that all too often such initiatives have failed to consider and appreciate the different levels at which racial discrimination operates and the multi-layered complexity of the sources of racialised inequalities. For example, Cunningham (Chapter 1) suggests that 'single-level' interventions such as those designed to effectuate individual behaviour change have failed to fully appreciate the complex and interconnected ways in which structural, organisational and individual factors play a role in perpetuating exclusionary practices. Similarly, other authors such as Heim, Corthouts and Scheerder (Chapter 3), Kilvington (Chapter 5) and McDonald and Spaaij (Chapter 6) have suggested that previous interventions have had little impact in challenging the structural arrangements 
of sporting cultures and organisations. Rankin-Wright and Hylton (Chapter 8) and Conricode and Bradbury (Chapter 13) extend this point further and argue that many interventions have been informed by 'liberal equal opportunities' approaches, which focus on increasing opportunities for under-represented minoritised groups whilst simultaneously leaving the conditions which perpetuate racialised inequalities relatively unchanged. As a result, there is a strong consensus amongst the authors featured in this collection that key stakeholders should broaden their focus and efforts to adopt a more broad ranging multi-level approach to addressing racialised inequalities in sports coaching. We provide concrete examples of recommended areas of focus and how these efforts might be undertaken in practice in each of the following three sections. Further, as has been argued by Rankin-Wright and Hylton (Chapter 8), Olushola-Ogunrinde and Carter-Francique (Chapter 9) and Knoppers and De Haan (Chapter 10) such an approach should also recognise, centralise and seek to redress the often overlooked intersectional forms of oppression and double burden of racialised and gendered discrimination experienced by minoritised women in sports coaching contexts. In this respect, any meaningful efforts to address racialised inequalities in sports coaching need to remain cognisant of the existence and expression of multiple intersectional forms of racism and discrimination, and the normative power of whiteness (and maleness) embedded in societal and sports coaching contexts. As has been argued by Bradbury (Chapter 2), Van Sterkenburg (Chapter 4) and Rankin-Wright and Hylton (Chapter 8), the tendency for key decision makers in sports to adhere to notions of meritocracy, race neutrality and colourblindness has tended to sustain rather than redress racialised inequalities in these settings. Further, Conricode and Bradbury (Chapter 13) suggest that liberalism alone is not enough to disrupt and dismantle these powerful processes and discourses, and a more strongly interventionist and transformational approach is needed to challenge and re-configure the long-standing structures and cultures that are regularly reproduced by those in positions of 
power within sports organisations. There is a strong consensus amongst the authors in this collection that a range of key stakeholders in sports - including, national governments, national sports federations, member sports organisations and clubs, equality bodies and campaign groups and minoritised coaches - should work collaboratively to develop and implement a more unified and holistic approach to addressing racialised inequalities in sports coaching. Such an approach should be appropriately resourced and engender a strong operational and attitudinal commitment to the principles and practice of diversity and inclusion at the structural, organisational and individual level. It should also utilise a range of contextually considered and targeted positive action measures to ensure equality of opportunities and experiences for minoritised coaches and to forcefully stimulate conditions under which equality of outcomes are more likely to be realised. We identify in more detail below three key areas of action that, when combined, can begin to provide this more holistic approach we have articulated above.

\section{(ii) Developing and implementing robust policy interventions}

Almost all of the authors in this collection have been explicit in their calls for key stakeholders and related bodies to work in consultation with minoritised coaches to develop and implement robust policy interventions designed to increase the representation of minoritised coaches and address racialised inequalities in sports coaching. In doing so, a number of authors such as Bradbury (Chapter 2) Heim, Corthouts and Scheerder (Chapter 3), and Van Sterkenburg (Chapter 4) have suggested that in the first instance this should include the establishment of clear policy goals and actions which incorporate the collection of baseline data, target setting, and the monitoring and evaluation of progress over time against clearly defined timescales. The allocation of roles and responsibilities for work of this kind 
should also reflect the organisational capacities, expertise and legal jurisdiction of key stakeholders and other bodies involved in this process.

As part of the aforementioned emphasis on policy development and implementation, there was a strong consensus amongst authors that key stakeholders and related bodies should undertake a more strongly regulated and interventionist approach to coach recruitment. Central to this discussion was the contribution of Duru (Chapter 11) which examined in detail the legislative development, operational implementation, and representational effectiveness of the 'Rooney Rule' in the US NFL. This positive action measure was championed by a number of authors (especially those examining racialised inequalities in football in Europe) as having significant transferability as an important consideration forcing mechanism through which to establish a more transparent and equitable approach to coach recruitment across a range of national and sporting contexts. In particular, in mandating that sports clubs must interview at least one suitably qualified candidate from a minoritised background for vacant senior coaching positions or incur a significant financial penalty. Further, some authors also alluded to the ways in which its operational focus, scope and effectiveness might be extended further. For example, both Bradbury (Chapter 2) and Cowell (Chapter 12) alluded to the importance of ensuring that measures of this kind adhere to and utilise creatively the opportunities embedded in national equalities legislation with regard to employment practices. Cowell (Chapter 12) also referenced the importance of consultation with local-level stakeholders and minoritised coaches at the design stage of such policy interventions, in order that their aims and intentions are better understood by those with direct responsibility for their enactment. Conricode and Bradbury (Chapter 13) argue further that such approaches are more likely to yield stronger operational and attitudinal buy-in to the principles and practice of racial inclusivity and engender more meaningful and sustainable organisational change. They continue that the provision of a uniform guidance document in relation to key 
components of newly formulated processes of coach recruitment might also help key decision makers to adhere to good equality practice in this respect. These points are extended further by Cunningham (Chapter 1) and Olushola-Ogunrinde and Carter-Francique (Chapter 9) who stress the importance of ensuring that the demographic make-up of selection and interview panels is culturally diverse and that appropriate feedback is provided to unsuccessful candidates to help them reflect on the interview process and better prepare for future applications. Further, Rankin-Wright and Hylton (Chapter 8), Olushola-Ogunrinde and Carter-Francique (Chapter 9), and Knoppers and De Haan (Chapter 10) suggest that positive action measures designed to diversify the sports coaching workforce should also pay attention to and seek to alleviate intersectional experiences of racialised and gendered exclusions and incorporate actions which consider multiple and multi-dimensional rather than binary forms of oppression. Finally, Duru (Chapter 11) draws attention to the likely increased representational impacts of extending the scope of the 'Rooney Rule' and related interventions to include a minimum of two candidates from under-represented groups. Such an approach is likely to increase opportunities for minoritised coaches from a broader range of racial, cultural, ethnic and gendered backgrounds to gain a foothold in the sports coaching industry.

A number of authors also called for regulatory and interventionist approaches to be extended beyond the coach recruitment process and into the field of coach education. For example, Conricode and Bradbury (Chapter 13) referenced positively the work of the English Football Association's Coach Inclusion and Diversity programme which provides financial and other resource support to empower minoritised coaches to achieve coaching qualifications and enhance their employability within the professional game. Other authors such as Heim, Corthouts and Scheerder (Chapter 3) argue that programmes of this kind should be rolled out in other national and sports coaching contexts (such as professional football in Belgium) and 
should include the provision of subsidised coach education and training programmes for aspiring minoritised coaches from socially and economically disadvantaged backgrounds. Van Sterkenburg (Chapter 4) extends this point further by suggesting that key stakeholder bodies should amend and broaden the selection criteria for elite level coaching courses such as the UEFA Pro-Licence in professional football in order to increase the appeal and accessibility of courses of this kind for talented minoritised coaches. Taken together, these and other authors in this collection suggested that the development and implementation of positive action interventions of this kind were necessary to unblock the early educational phase of the pipeline of progression in sports coaching and to empower minoritised coaches with the requisite skill sets and confidence to proceed into the professional coaching environs of sports.

\section{(iii) Developing and implementing education and training programmes}

A large proportion of the authors in this collection have suggested the need for the delivery of education programmes targeted at influential stakeholders in sports coaching, particularly those involved in the process of coach education and coach recruitment. For many authors, these personnel have acted as key gatekeepers with the powers to grant or withhold access to the developmental and occupational marketplace of sports coaching and have played a pivotal role in sustaining patterns of homologous reproduction within this realm. Further, as Conricode and Bradbury (Chapter 13) have indicated, these personnel often display a strong adherence to notions of meritocracy, race neutrality and colour-blind ideologies and consequently their understanding of processes of racialisation and racialised inequalities in sports coaching remains limited. In this respect, authors such as Bradbury (Chapter 2), Heim, Corthouts and Scheerder (Chapter 3), and Kilvington (Chapter 5) have argued that educational activities should pay particular attention to the ways in which overt, culturally 
coded and more institutional forms of racism and discrimination can work at a conscious and unconscious level to constrain the opportunities of and engender negative experiences for minoritised coaches. Rankin-Wright and Hylton (Chapter 8) and Olushola-Ogunrinde and Carter-Francique (Chapter 9) extend this point further and suggest that a much greater awareness of the interconnections between systems of oppression is needed to enable key stakeholders to understand and address racialised and gendered exclusions in sports coaching settings.

For authors such as McDonald and Spaaij (Chapter 6) pedagogical approaches of this kind should also seek to challenge and debunk stereotypical ideas and practices around the racialised black body and related deficit discourses which unproblematically position black athletes and coaches as lacking the requisite psychological characteristics to succeed in decision-making roles in sports. Similarly, Light and Evans (Chapter 7) suggest that coaches should think more strongly and reflexively as to the racialised nature of dominant antidialogic coaching practices and exhibit greater cultural sensitivity and understanding in their dealings with minoritised athletes. Taken together, these authors suggest that coaches and sports science professionals should engage in formalised educational activities and informal pedagogical reflection in order to challenge the everyday racialised assumptions and patterns and processes of White privilege embedded within such environs. Van Sterkenburg (Chapter 4) extends this discussion further to suggest that more educational work is needed to challenge racial stereotyping within the sport media given the powerful role they play in constructing and reproducing racialised narratives and discourse and framing popular debates around the suitability of minoritised coaches for appointments in elite level sports settings. Conricode and Bradbury (Chapter 13) have argued that educational programmes targeting senior power brokers at professional sports clubs should also be utilised as a mechanism through which to engender stronger operational and attitudinal 'buy-in' to emerging policy 
interventions designed to increase the representation of minoritised coaches. This assertion chimes strongly with the points made previously about the need for a holistically considered multi-level approach to addressing racialised inequalities in sports coaching. In this respect, such programmes should contextualise the relevance and applicability of policy interventions to the sports coaching settings in which they are being implemented. They should also encourage a perceptual shift in conceptualising cultural diversity less as a problem to be 'fixed' or 'dealt with', and more as a positive individual and organisational resource to be nurtured and developed. In doing so, the authors argue that pedagogical work of this kind should feature a strong emphasis on increasing understanding of the potential and realised benefits of cultural diversity as means through which to enhance organisational effectiveness in the coaching workplace. Taken together, the calls for the development of education and training programmes by authors in this collection share a common theme in engendering an analytical shift away from a singular (albeit important) focus on 'upskilling' excluded participants, towards a more transformational approach which seeks to challenge and change the institutionally closed structures and cultures of sports organisations and the processes and discourses of whiteness which underpin them.

\section{(iv) Enhancing networks, social capital and opportunities for minoritised coaches}

Finally, a number of authors in this collection have argued that establishing, maintaining and utilising contacts and relationships with key 'insider' figures is an important source of social capital that has been shown to have significant influence on the development, recruitment and promotion of sports coaches. This is especially the case in relation to the elite level environs of coach education and coach employment where the over-reliance on networks-based approaches to identification, selection and recruitment has traditionally favoured White coaches drawn from within the dominant social and cultural insider networks of the sport 
industry. As a result, one of the most common recommendations for practice from authors was the need to strengthen and broaden the networks available to coaches from minoritised backgrounds. In this respect, Kilvington (Chapter 5) has called for more efforts to develop networks that encourage both 'bonding' forms of social capital where those from similar backgrounds can strengthen ties and share experiences, and 'bridging' social capital where networks are created to bring together people and groups who might otherwise rarely connect (Putnam 2000). Kilvington (Chapter 5) continues that more needs to be done by sporting authorities to facilitate and expand upon existing but poorly resourced physical and online networks which bring together minoritised coaches and key stakeholders. Such networks are argued to provide a useful interactional space for minoritised coaches to learn about and access support to meet key developmental and organisational requirements and for key stakeholders to increase their understanding of key cultural considerations which might impact on the pace and scope of career progression.

Olushola-Ogunrinde and Carter-Francique (Chapter 9) extend this latter discussion further in drawing attention to the potential benefits of establishing formal and informal mentoring and advocacy programmes which can support the growth and development and raise the profile of minoritised coaches in sports coaching contexts. Relatedly, Van Sterkenburg (Chapter 4) has suggested that national football associations (and by proxy, sports organisations more broadly) should establish a national database of all minoritised coaches in receipt of elite level coaching qualifications. This 'ready list' of highly qualified minoritised coaches could be circulated to professional clubs as well as being used as a mechanism through which to alert minoritised coaches to emergent coaching positions in their respective sports. Such an approach would increase the awareness of and applications for coaching opportunities in sports and increase the visibility of minoritised coaches within the eyeline and consciousness of power brokers within sports clubs. Taken together, efforts of this kind are likely to yield 
some success in increasing the network capital of minoritised coaches, broadening the previously closed processes of coach recruitment at sports clubs, and diversifying the sports coaching workforce.

\section{Concluding remarks}

Whilst in this final chapter we have discussed quite separately three key areas of future research priority - macro, meso and micro - and then four sets of recommendations, they should not be seen in isolation from one another. The point has been made several times that a holistic and multi-level approach is called for to be able to research and then design interventions that have the potential to create meaningful and long lasting change. The example of how we might go about changing recruitment practices illustrates this holistic and multi-level approach well. Taken together, the chapters featured in this collection point to the need for a multi-pronged approach that requires interventions across several layers. The historic recruitment practices that have dominated sports organisations need dismantling through structural interventions such as the 'Rooney Rule' and other forms of mandatory positive action schemes that have the potential to disrupt the reproduction of enduring forms of racialised privilege. Enforced changes to the membership of recruitment panels may also help encourage more equitable outcomes in the hiring of coaches. More deeply considered, contextually relevant and pedagogically effective education/training for those with responsibility for coach recruitment is also needed. This should be accompanied by activities that will develop networks to enable minoritised coaches to access the existing structures and cultures of coaching and become 'legitimate' candidates for recruitment.

The adoption of a somewhat 'radical' policy such as the 'Rooney Rule' illustrates not only how a field such as sport can be disrupted by anti-racist politics and critical academic thought, but that sport can play a central role in raising the anti-racist agenda across other 
spheres of social life. In this respect, sport, and specifically sports coaching, offers us a crucial site from which to embed the message of racial equality and find new ways of disrupting racialised forms of discrimination across a range of contemporary national contexts. The weight of empirical evidence backs up the casual observations of sports enthusiasts in that many minoritised athletes are not transitioning into elite coaching positions. This gives us legitimacy to continue to document such disparities, publicise them to both sports organisations and the mainstream media, and to critically evaluate the strengths and limitations of initiatives designed to address racialised inequalities in sports coaching. Many of us probably feel that in recent times we have gone significantly backwards in the fight for racial equality across sport and wider society. Some of the 'gains' we might have reasonably claimed to have made over the last 20 years of anti-racist politics and critical scholarly endeavour seem to be increasingly under threat from a new hostile political environment. In this rapidly changing social context, the popular appeal of sport has arguably never been a more important site to challenge racialised and other forms of discrimination and exclusion. We hope this collection inspires readers to continue to pursue research and implement interventions that aim to diversify the coaching workforce, finding creative ways to dismantle the racialised inequalities and barriers that still inform people's opportunities, experiences and outcomes in sports coaching.

\section{References}

Ahmed, S. (2012) On Being Included: Racism and Diversity in Institutional Life. Durham, NC: Duke University Press.

Ahmed, S. (2007) The language of diversity. Ethnic and Racial Studies, 30(2), 235-256. Bonilla-Silva, E. (2015) The structure of racism in color-blind, 'post-racial' America. American Behavioral Scientist, 59(11), 1358-1376.

Bonilla-Silva, E. (2010) Racism Without Racists: Colour-blind Racism and the Persistence of Racial Equality in the United States (Third Edition). Oxford: Rowman and Littlefield. Boykoff, J. and Carrington, B. (2019) Sporting dissent: Colin Kaepernick, NFL activism, and media framing contests. International Review for the Sociology of Sport [online].

DOI:1012690219861594.

Burdsey, D. (2014) One week in October: Luis Suárez, John Terry and the turn to racial neoliberalism in English men's professional football. Identities, 21(5), 429-447. 
Bury, J. (2015) Non-performing inclusion: A critique of the English Football Association's Action Plan on homophobia in football. International Review for the Sociology of Sport, 50(2), 211-226.

Cabrera, N. L. (2018) Where is the Racial Theory in Critical Race Theory? A constructive criticism of the Crits. The Review of Higher Education, 42(1), 209-233.

Cabrera, N. L., Franklin, J. D. and Watson, J. S. (2017) Whiteness in Higher Education: The Invisible Missing Link in Diversity and Racial Analyses. Association for the Study of Higher Education monograph series. San Francisco, CA: Jossey-Bass.

Castells, M. (1997) An introduction to the information age. City, 2(7), 6-16.

Cole, M. (2017) Critical Race Theory and Education: A Marxist Response. London: Springer.

Delgado, R. and Stefancic, J. (2012) Critical Race Theory: An Introduction (Second Edition). London: NYU Press.

Doherty, A., Fink, J. and Inglis, S. (2010) Understanding a culture of diversity through frameworks of power and change. Sport Management Review, 13, 368-381.

Dwight, A. and Biscomb, K. (2018) Ten years of the UK's Equality Standard for Sport. European Journal for Sport and Society, 15(2), 171-188.

Fukuyama, F. (2006) The End of History and the Last Man. London: Simon and Schuster.

Gearity, B. and Henderson Metzger, L. (2017) Intersectionality, micro-aggressions and micro-affirmations: Towards a cultural praxis of sports coaching. Sociology of Sport Journal. 34(2), 160-175.

Giroux, H. (1997) Pedagogy and the Politics of Hope: Theory, Culture and Schooling: A Critical Reader. Boulder: Westview.

Glathe, J. (2016) Football fan subculture in Russia: Aggressive support, readiness to fight, and far right links. Europe-Asia Studies, 68(9), 1506-1525.

Goldberg, D. T. (2009) The Threat of Race: Reflections on Racial Neoliberalism. Chichester: Wiley Press.

Hylton, K. (2005) 'Race', sport and leisure: Lessons from critical race theory. Leisure Studies, 24(1), 81-98.

Hylton, K. (2009) 'Race' and Sport: Critical Race Theory. London: Routledge.

Hylton, K. (2010) How a turn to critical race theory can contribute to our understanding of 'race', racism and anti-racism in sport. International Review for the Sociology of Sport, 45(3), 335-354.

Hylton, K. (2015) 'Race' talk! Tensions and contradictions in sport and PE. Physical Education and Sport Pedagogy, 20(5), 503-516.

Kapoor, N. (2013) The advancement of racial neoliberalism in Britain. Ethnic and Racial Studies, 36(6), 1028-1046.

Farrington, N., Hall, L., Kilvington, D., Price, J., and Saeed, A. (2015) Sport, Racism and Social Media. London: Routledge.

Lusted, J. (2017) Understanding the varied responses to calls for a 'Rooney rule' in English football. In D. Kilvington and J. Price (eds.) Sport and Discrimination. London: Routledge, 44-57.

Lusted, J. (2013) Selling race equality to sport organisations: Challenges and limitations. In D. Hassan and J. Lusted (eds), Managing Sport: Social and Cultural Perspectives. London: Routledge, 90-107.

Noon, M. (2007) The fatal flaws of diversity and the business case for ethnic minorities. Work, Employment and Society, 21(4), 773-784.

Putnam, R. D. (2000) Bowling Alone: The Collapse and Revival of American Community. London: Simon and Schuster. 
Solórzano, D. G. and Yosso, T. J. (2002) Critical race methodology: Counter-storytelling as an analytical framework for education research. Qualitative Inquiry, 8(1), 23-44.

Shaw, S. (2007) Touching the intangible? An analysis of the equality standard: A framework for sport. Equal Opportunities International, 26(5), 420-434.

Spaaij, R., Knoppers, A. and Jeanes, R. (2019) 'We want more diversity but...': Resisting diversity in recreational sports clubs. Sport Management Review [online] - available at https://doi.org/10.1016/j.smr.2019.05.007

Spaaij, R., Magee, J., Farquharson, K., Gorman, S., Jeanes, R., Lusher, D. and Storr, R. (2018) Diversity work in community sport organizations: Commitment, resistance and institutional change. International Review for the Sociology of Sport, 53(3), 278-295. Spracklen, K., Hylton, K. and Long, J. (2006) Managing and monitoring equality and diversity in UK sport: An evaluation of the sporting equals racial equality standard and its impact on organizational change. Journal of Sport and Social Issues, 30(3), 289-305. Testa, A. and Armstrong, G. (2010) Football, Fascism and Fandom: The Ultras of Italian Football. London: A\&C Black. 